\title{
Weitere Beiträge zur Kenntnis der Kombination von tertiär- luetischer, cerebraler Erkrankung mit progressiver Paralyse und über Erweichungsherde bei Paralyse.
}

\author{
Von \\ Priv.-Doz. Dr. Ernst Sträussler, \\ k, und k. Regimentsarzt. \\ (Aus der deutschen psychiatrischen Universitätsklinik in Prag.) \\ Mit 1 Textfigur und I Tafel. \\ (Eingegangen am 11. Juli 1912.)
}

Meine Publikation von 4 Fällen von Kombination cerebraler, tertiärer, gummöser Lues mit progressiver Paralyse ${ }^{1}$ ) hat bisher nicht den erwarteten Widerhall in Fachkreisen geweckt. Die Erwartung, daß unsere Beobachtungen von miliaren Gummen die Anregung zur Veröffentlichung ähnlicher Befunde von anderer Seite geben würden, hat sich nur in einem Falle erfültt. Landsberge ${ }^{2}$ ) beschreibt eine Beobachtung von miliaren Gummen in der Hirnrinde und in den Stammganglien, während die von Witte ${ }^{3}$ ) und von Giljarowsky ${ }^{4}$ ) in je einem Falle von Paralyse geschilderten, und der echten Syphilis zugerechneten Veränderungen nicht den Charakter g u m möser Prozesse aufweisen.

Wenn wir bedenken, daß die von uns veröffentlichten 4 Fälle von gummöser, zerebraler Lues auf 130 genauer untersuchte Paralysen fielen, was, bei der relativen Seltenheit von gummösen Prozessen im Gehirn überhaupt, ein überraschend großes prozentuelles Verhältnis darstellt, so können wir den etwa auftauchenden Gedanken, daß dieses Ergebnis dem Walten eines Zufalles in unserem Materiale zuzuschreiben wäre, von der Hand weisen; wir müssen vielmehr annehmen, daß eine entsprechende Nachforschung in einem anderen Paralytikermateriale ein ähnliches Resultat zeitigen müßte.

1) Monatsschr. f. Psych. u. Neurol. 19, 1906 u. 2g, 1910.

2) Lues cerebri und progressive Paralyse, ein klinischer und anatomischer Beitrag. Monatsschr. f. Psych. u. Neurol. 29. 1911.

3) Über eine eigenartige herdförmige Gefäßerkrankung bei Dementia paralytica. Diese Zeitschr. 2. 1910.

4) Ein anatomischer Beitrag zur Frage über die Beziehungen der progressiven Paralyse zu der Gehirnsyphilis. Diese Zeitschr. 6. 1911. 
Wir sind um so mehr berechtigt, diese Anschauung zu vertreten, als wir in der Lage sind, wieder über zwei ne ue Fälle von cerebraler tertiärer Lues bei progressiver Paralyse zu berichten. Sie stammen aus 40 Fällen der fortgesetzten Reihe von histologischen Untersuchungen der an der Klinik zum Exitus gelangten Paralysen, und wir verfügen also gegenwärtig über 6 Beobachtungen von tertiärluetischer cerebraler Erkrankung in einem Materiale von 170 Paralysen.

Von den beiden neuen Beobachtungen handelt es sich in dem einen Falle um eine luetische Konvexitätsmeningitis bei einer juvenilen Paralyse, im anderen um miliare Gummen in der Hirnsubstanz; gegenüber den bisher publizierten Beobachtungen von miliaren Gummen bietet dieser Kasus die Besonderheit, daß die syphilitischen Herdchen nicht auf die Rinde beschränkt bleiben, sondern a u ch über die der Rinde angrenzende Marks ubstanz verstreut sind.

Der Fall ist aber noch durch einen anderen bemerkenswerten Befund ausgezeichnet: es besteht neben kleinen älteren Encephalomalacien eine große frische Erweichung im linken Linsenkern.

Halten wir uns vor Augen, daß die Erkrankung an Paralyse wohl ausnahmslos auf einer vorangegangenen luetischen Infektion beruht, so wäre von vornherein anzunehmen, daß eine Komplikation der progressiven Paralyse mit Encephalomalacien zu den alltäglichen Erscheinungen gehöre. Bildet doch die Lues recht häufig den Anlaß zur Entstehung von Encephalomalacien in allen Lebensaltern infolge He ubnerscher Endarteritis; anderseits gilt es als feststehende Tatsache, da $\beta$ die luetische Erkrankung die Entwicklung der Arteriosklerose in hohem Maßse befördert und also auch auf diesem Wege zu Erweichungen bei progressiver Paralyse führen könnte.

In meiner Abhandlung über die histopathologischen Veränderungen des Kleinhirns bei der progressiven Paralyse ${ }^{1}$ ) habe ich mich mit der Frage der luetischen Gefä ßerkrankung bei progressiver Paralyse des Näheren beschäftigt und auf die hinsichtlich der Häufigkeit syphilitischer Veränderungen der Gefäße bei Paralyse in den Literaturangaben bestehenden großen Differenzen hingewiesen. In meinem eigenen, damals verarbeiteten Paralytikermateriale konnte ich in $13,5 \%$ der Fälle eine Gefäßerkrankung nachweisen, welcher die Charaktere einer luetischen Affektion zugesprochen werden mußten.

Jedenfalls steht es fest, daß das Vorkommen der He ubnerschen Erkrankung an den Gehirngefäßen bei progressiver Paralyse keine Seltenheit ist; zu den von uns nachgewiesenen zweifellos echten syphili-

1) Jahrbücher f. Psych. u. Neurol. 27. 1906. 
tischen Erscheinungen bei Paralyse bilden diese Befunde an den Gefäßen eine natürliche Ergänzung.

Angesichts dieser Umstände muß man sich wundern, daß Encephalomalacien in der pathologischen Anatomie der progressiven Paralyse kaum eine Erwähnung finden. Man entdeckt wohl in der Literatur einzelne Fälle, in welchen von, meist alten, Erweichungsherden so von ungefähr die Rede ist; aber weder in den Lehrbüchern noch in den zahlreichen Monographien über progressive Paralyse, bis auf Mende ${ }^{\mathbf{1}}$ ), erscheint das Vorkommen von Encephalomalacien auch nur mit einem Worte gew ürdigt.

Die erwähnte Beobachtung, in welcher der progressiven Paralyse einerseits syphilitische Erseheinungen und anderseits encephalomalacische Prozesse zugesellt waren, veranlaßte uns das Paralytikermaterial, welchem die 6 Fälle von luetischer Gehirnerkrankung entstammen, hinsichtlich etwa vorhandener Erweichungsherde zu kontrollieren, und es zeigte sich, daß noch drei weitere Fälle von progressiver Paralyse mit Encephalomalacien kompliziert waren. Wenn das auch keinen besonders großen Prozentsatz bedeutet, so erscheinen uns die Befunde für die pathologische Anatomie und die Klinik der progressiven Paralyse doch von genügender Wichtigkeit, um sie einer entsprechenden Würdigung für wert zu erachten.

Zunächst soll hier die Beschreibung der luetischen Konvexitätsmeningitis bei einer juvenilen Paralyse Raum finden.

I. Das 14jährige Bauernmädchen B. M. wurde am 15 . Mai 1908 in die Klinik aufgenommen.

Ihre Stiefmutter, wolche die Pat. als $4^{1 / 2}$ jähriges Kind in Pflege bekan, gibt zur Anamnese an, daß sie in der Schule schlecht gelernt, schließlich aber doch genügende Fertigkeit im Schreiben und Lesen erlangt habe. Sie sei immer mehr in sich gekehrt und sehr träge gewesen.

Endedes Winters 1907 habe sich plötzlich in ihrem Wesen eine a uffallende Wandlung vollzogen, sic sei frech und sehr gesprächig geworden, wobei sic vicl unsinniges Ze ug vorgebracht und sich obscöner Redensarten bedient hätte. Mehrmals soi sie rom Hanse fortyclaufen, narkt auf die Gasse gegangen, und einmal hitbe sie ihre kleine htiefsehwester grebissen, als sie mit ihr allein zu Hause gelassen wurde.

Hinwichtlich hetis her Erscheinungen der Pat. scllost, sowie einer luetischen Erkrankung in deren Ascendenz ist nichts bekannt.

Die Cntersuchang der Pat. bei der Aufnahme eroibt: Im Wachstum zurïekgeblieben, kindlieher Habitus, Geuitale noch vollstandig haarlos. Schädel relativ groß, Unfang $51,5 \mathrm{~cm}$, starke Vortreibung der T'ubera frontalia. Ausgesprochene sattelnase. Starke Skoliose der mittleren Brustwirbelsäule and humbale Lordose. Die Otwersehenkel nach außen verkrümmt. Genua valga.

Pupillen different, die rechte exzentrisch gelagert und enger als die linke, die linke Pupille absolut starr (l,icht- und Konvergenzreaktion fehlend), die rechte

1) Mendel, Die progressive Paralyse der Irren 1880. M. zitiert aus der Literatur 4 Fäle von bei Paralyse beobachteten dirweichungen. 
reagiert ziemlich gut. Facialis rechts besser innerviert als links, die $Z$ unge weicht nach rechts $a b$. Kniephänomen hochgradig gesteigert, beiderseits FuBklonus, rechts Andeutung von Babinski.

In psychischer Hinsicht fällt die Pat. durch ein nicht ihrem Alter angepaßtes kindisches Wesen auf, äußert, sie werde sich ein Portemonnaie und für 10 Kreuzer Kleider, Schuhe und ein Pferd kaufen. Sie ist sehr vergnügt und singt verschiedencrlei Lieder mit ganz falscher Melodie, sagt Gedichte auf. Dann erzählt sie mit heiterer Miene, daß ihr Vater heute gestorben sei und auf dem neuen Friedhof begraben werde; lacht ganz seelenvergnügt dazu, meint aber dann, sie werde weinen, ,alle weinen, wenn jemand stirbt". Sie sei hergekommen, nachdem sie von ihrer Mutter aus dem Hause gejagt worden wäre; hier gefalle es ihr gut.

Sie erkennt viele der gangbarsten Münzsorten nicht, schreibt fehlerhaft, bei einer Leseprobe liest sie langsam, fehlerhaft, jedes Wort einzeln, ohne Verständnis. Über Zeit und Ort weiß sie keinen richtigen Bescheid zu geben. Auch noch nach mehrtägigem Aufenthalte in der Klinik hat sie sich örtlich nicht orientiert.

Im Laufe der nächsten Monate macht ihre Lustigkeit und Redseligkeit allmählich einem stillen, stumpfen Wesen Platz. Sie arbeitet gar nichts, sitzt den ganzen Tag auf einem Platze dort, wo man sie hingesetzt hat; sie achtet auf ihre körperliche Reinlichkeit gar nicht, mitunter benäßt sie sich. Die Sprache wird verwaschen, hin und wieder tritt Silbenstolpern deutlich zutage.

Eine hochgradige stumpfe Demenz steht dann im weiteren Verlaufe der Krankheit im Vordergrund des klinischen Bildes, bis Anfang September 1909 Aufregungszustände auftreten, in welchen dic Kranke abwechselnd lacht und weint, in die Bettdecke beißt, schreit und heult. In den letzten Tagen des Monates Oktober 1909 tritt bei Fortdauer der Erregung ein kontinuierliches Fieber auf, welches sich um $39,0^{\circ}$ bewegt. Am 2. November wird ein Anfall beobachtet mit Wendung des Kopfes und der Augen nach links und Zuckungen in der rechten Hand. Die Hand zeigt dann den ganzen Tag über ein grobes Zittern, der Kopf bleibt nach links gewendet, es besteht Nackensteifigkeit, Benommenheit. Am nächsten Tage wiederholen sich die Anfälle, es sind Erscheinungen einer Pneumonie nachweisbar. Die Untersuchung der Cerebrospinalflüssigkeit ergibt 700 Zellen im cmm. Am 4. November 1909 tritt der Exitus ein.

Aus dem allgemeinen Sektionsbef $u$ nd ist zu erwähnen, daß sich an der Zwerchfellfläche der Leber mehrere kleine circumseripte Einziehungen befinden und daß das Genitale, Uterus und Vagina, durch einen infantilen Charakter ausgezeichnet ist.

Bei der Inspektion des Gehirns fällt eine starke Trübung und Verdickung der Meningen in die Augen. Die Gehirnfurchen sind an der Convexität durch die verdickten Meningen überbrïckt, so daß3 stellenweise eine fast gleichmäßige Nivellierung der Gehirnoberfläche zustande kommt. Die Affektion der meningealen Bedeckung betrifft in besonders starkem Maße die rechte Hemisphäre, aber auch hier zeigt die Intensität der Krkrankung an verschiedenen Orten ziemlich bedeutende Schwankungen. In der Gegend des unteren Teiles der Zentralwindungen ind in Berciche des Schläfelappens erreicht die Trübung und Verdickung der Meningen die höchsten Grade, sie stellen eine grauweiße, fellartige Membran dar.

Die Affektion der Meningen erstreckt sich auch aut die Gehirnbasis, und hier erreicht der Prozeß seinen Höhepunkt in der Gegend des Chiasma nervi optici.

Das Gehirn wurde in toto der Formolhärtung unterzogen.

Fin durch das gehärtete Gehirn geführter Horizontalschnitt, welcher die Seitenventrikel eröffnet, zeigt, daß diese in beiden Hemisphären bedeutend 
erweitert sind; dabei betrifft die Frweiterung in unverhältnismäßig starkem Maße die Vorderhörner. Die der rechten Hemisphäre zugehörige Schnittfläche zeigt gleichzeitig, daß die Sylvische furche durch eine weißlich-graue, sulzige Masse ausgregossen ist; zwisehen der Insel und dem Opercularteile des unteren Scheitelläppchens erreicht der Ausguß an manchen Stellen Zentimeterdicke und sendet schwächere Ausläufer in alle benachbarten Furchen hincin.

Das Kleinhirn bictet hinsichtlich seiner Größe keine Auffälligkeit; die Meningen sind in geringerem Grade getrübt und verdickt, am stärksten über dem Vermis superior. An der unteren Fläche der rechten Hemisphäre ist die Gehirnsubstanz in eines kleinen, rtwa ${ }_{2}^{1:} \mathrm{cm}$ im Durchmesser haltenden Stelle weißlich getrübt und die in den Meningen ziehenden Gefäßse scheinen über dem Herdehen eine Unterbrechung zu arfahren. Die Hirnsubstanz fühlt sich an dieser Stelle derber an, und am Durchschnitt sieht man einerseits, da $B$ dic Gefäße der Meningen in solide Stränge umgewandelt sind und andersits, dâs die Kleinhirnlippohen eine homogene grau-weibe Färbing darbieten, indem die durch die Farbdifferenz zwischen Rinde und Mark entstehende charakteristische Zeichnung vollkommen verwischt ist.

Dir Meningen des Pons und der Medulla oblongata zeigen nur eine geringe Trübung, die sich auch noch auf dic meningeale Bedeckung des Riicken marks fortsetzt. Die basalen Hirngefäßc erscheinen bei makroskopischer Betrarhtung zirt.

Mikroskopische Untersuchung: Am Großhirn interessieren uns nach den Krgebnissen der makroskopischen Untersuchung vor allem die Meningen. Nehmen wir zunächst einen Schnitt aus dem Stirnhirn vor, welcher derart gewählt ist, daß wir an ihm verschiedene Grade der meningealen Veränderung studieren können, so ergeben sich beim Verschieben des Präparates unter dem Mikroskop recht wechselnde Bilder:

1. Kine mäBig starke Verbreiterung der meningealen Bedeckung des Gehirns, mit deutlicher Scheidung in 2 Schichten: Die dem Gehirn benachbarten Blätter mit starker Infiltration heben sich auffallend von dem oberflächlichen Anteil der Meningen ab, in welchen Bindegewebsfasern die Oberhand besitzen und das mikroskopische Bild bestimmen. Die Infiltration der visceralen Partien der Meningen besteht aus meist großen, vielfach in Regression befindlichen Plasmazellen; das Gebiet der Bindegewebsproliferation zeichnet sich durch die große Zahl succulenter Fibroblasten aus. Diese Schichte ist aber nicht ganz frei von Infiltrationszellen; hin und wieder sind es typische Plas mazellen, meist entfernen sie sich aber mehr oder weniger von der gewöhnlichen Form dieser Infiltrationszellen, der Protoplasmaleib gewinnt eine größere Ausdehnung und eine runde Form. Es sind die gleichen Zellen, wie sie Schaffer in seinem Falle von "Pseudoparalysis syphilitica" (Seite 212,213 u. Fig. 7, 2) ${ }^{1}$ ) beschrieben hatte. Ich schließe mich seiner Deutung dieser Zellen als Plasmazellen an.

2. Die Meningen erlangen eine größere Breite, einerseits durch Wachsen der Infiltrationszone und anderseits durch Zunahme der Binde-

1) Zeitschr. f. d. ges. Neurol. u. Psych. 3. 1910.

z. f. a. g. Neur. u. Psych. O. XII. 
gewebswucherung. Die Infiltrationszellen sind nun auch viel dichter in den visceralen Partien der Meningen angeordnet als früher (Taf. XI, Fig. 1), und sie tragen viel häufiger das Gepräge junger Formen. Die Bindegewebsschichte ist durch zahlreiche junge, zarte Bindegewebsbälkchen ausgezeichnet, zwischen welchen homogene Massen (Gerinnsel) eingeschoben sind. Die früher beschriebenen runden Zellen haben hier an Zahl zugenommen und zeigen einerseits Erscheinungen lebhafter Proliferationsvorgänge, anderseits Symptome von degenerativen Prozessen. Viele dieser Zellen weisen 2 Kerne auf, zuweilen findet man 3, 4 und 5 Kerne; in anderen Zellen erfährt das Protoplasma eine Vacuolisation, hin und wieder treten in einer Zelle mehrere Vacuolen auf, die mitunter zusammenfließen, so daß schließlich die Zelle nur mehr durch den dunkeln Kontur der Umgebung gegenüber kenntlich bleibt.

3. Die Schichte der Infiltrationszellen gewinnt ein verändertes Bild durch Auftreten von zahlreichen Lymphocyten; die Gesamtdicke der Meningen nimmt gleichzeitig weiter zu; in den oberflächlichen Schichten sind noch die großen Zellen sichtbar.

4. Die sehr stark verdickten Meningen sind in ihrer ganzen Breite von dicht angeordneten L y m phoc yte n durchsetzt; die zwei Schichten existieren nicht mehr. An manchen Stellen ist die durch Kerne der Lymphocyten bedingte dunkle Färbung des Gewebes durch blaß bläulich tingierte Flecken unterbrochen, und die Untersuchung mit stärkeren Linsen ergibt, daß hier nekrotische Prozesse platzgegriffen haben; man sieht Detritusmassen und epitheloide Zellen. An manchen Orten findet man homogen glasige, von feinen Fibrinfasern durchzogene Exsudatmassen.

Dieser schematischen Schilderung ist wohl hinzuzufügen, daß die hier beschriebenen verschiedenen Stufen des Prozesses keineswegs scharf voneinander getrennt sind; es kommen die mannigfachsten Kombinationen und Übergänge vor.

Wir haben in dieser Schilderung noch nicht der Gefä Be gedacht, deren Verhalten einer entsprechenden Würdigung bedarf. Sie zeigen sich fast überall in hohem Grade affiziert: die Gefäßwandungen sind von Infiltrationszellen, teils Lymphocyten, teils Plasmazellen durchsetzt, und zwar erscheint die Adventitia in besonders hohem Grade von dieser Veränderung betroffen. Meist zeigt aber auch die Intima einen sehr auffallenden Befund: sowohl in Arterien wie auch Venen sieht man eine lebhafte Proliferation und Infiltration, so daß in großen Gefäßen sehr zellreiche, polsterförmige Auflagerungen entstehen, in kleinen das Lumen oft auf ein Minimum eingeengt wird. In den der Nekrose verfallenen Partien der Meningen findet man dann Gebilde, welche als obliterierte Gefäße imponieren, indem noch cirkulär angeordnete Bindegewebszüge und Endothelzellen die Gefäßwand andeuten, während das 
Lumen durch eine in ihrer Struktur verwischte Masse ausgefüllt erscheint.

Bemerkenswert ist die Häufigkeit des Befundes von Mastzellen, welche sich ziemlich oft an die Gefäße anschließen.

Die grauen, sulzigen Massen, welche in die Fissura Sylvii eingelagert sind, zeigen sich im mikroskopischen Schnitte unter folgendem Bilde: sozusagen die Stützsubstanz der pathologischen Bildung wird im wesentlichen durch die vermehrten und krankhaft veränderten Gefäße dargestellt. Deren Wände sind überschwemmt von Infiltrationszellen, unter welchen Lymphocyten die Oberhand besitzen; die Struktur der Gefäßwände geht in den massigen Infiltraten unter; die Fndothelwucherungen erreichen hier besonders hohe Grade. Im Anschlusse an die Gefäße haben sich breite, weit in die Umgebung hineinreichende Infiltrationsringe gebildet. Zwischen die Gefäße und die ebenfalls infiltrierten Bindegewebsfasern der Meningen sind homogene, bei Methylenblaufärbung leicht grünlich, durch Eosin blaß bis dunkelrosa gefärbte Exsudatmassen eingelagert.

Wenden wir uns der Schilderung des mikroskopischen Hirnbef undes zu, so haben wir uns zunächst mit den unmittelbaren Folgen der meningealen Affektion für die Hirnsubstanz zu beschäftigen.

Die günstigsten Bedingungen für dieses Studium bietet die Inselgegend, in welcher die meningealen Veränderungen die höchsten Grade erreichen; hier ist auch die Hirnsubstanz am stärksten in Mitleidenschaft gezogen.

An vielen Stellen findet eine so innige Versch melz ung zwischen Meningen und Hirnsubstanz statt, daß die Grenzen kaum mehr sicherzustellen sind; dies trifft in besonderem Maße dort zu, wo die an das Gehirn angrenzenden Schichten der Meningen von ne krotischen Prozessen heimgesucht wurden. Die Ernährungsstörung, welche durch die Gefäßerkrankung bedingt, das meningeale Gewebe in eine trübe, strukturarme Masse verwande t hat, macht sich auch in den oberflächlichen Rindenschichten geltend, auch hier erscheint die normale Gewebsstruktur verwischt. I)ie Reaktionsfähigkeit der Glia tritt in ihre Rechte, eine lebhafte Wucherung des gliösen Ge we bes versorgt die betroffene Hirnpartie mit massenhaften Gliazellen und Fasern, welche sich nicht selten in Form eines Walles vor das tiefere Hirngewebe lagern. (Tafel XI, Fig. 2.)

Dort wo der viscerale Anteil der Meningen von massigen Lymphocyteninfiltrationen eingenommen ist, findet die Beeinflussung der obersten Hirnpartien in der Weise statt, daß an manchen Stellen eine Überschwemmung des angrenzenden Hirngewebes, der obersten Rindenschichten, mit Lymphocyten platzgreift; meist dringen die Entzündungselemente sichtlich im Anschluß an die Gefäße in die Tiefe, nicht selten 
findet man sie aber auch frei im Gewebe. Hin und wieder häufen sich die Infiltrationszellen in einem umgrenzten Gebiete in Form eines miliaren Herdes an. Auch hier findet eine lebhafte Gliawucherung statt.

Im Bereiche einer derartigen Beeinflussung des Hirngewebes findet sich bis in die tiefsten Rindenschichten, ja auch bis in die Marksubstanz hinein eine lebhafte Gefäßwucherung; alle Gefäße weisen eine starke Infiltration ihrer Wände auf, nur an wenigen Orten ist die Infiltration eine vornehmlich lymphocytäre, die große Mehrzahl der Gefäße ist von Plasmazellen eingesäumt; hin und wieder treten auch die früher beschriebenen großen Formen auf, welche sich in den oberflächlichen Schichten der Meningen in so großer Zahl finden. Neben der Gefä $\beta w$ ucherung, den Infiltrationen und einer sehr deutlichen $W$ ucherung der Gefäßendothelien, fesseln noch die proliferativen Vorgänge der Glia mit zahlreichen Spinnenzellen und Gliarasen, und die große Zahl von Stäbchenzellen in besonderem Maße die Aufmerksamkeit.

Was das nervöse Gewebe betrifft, so bemerkt man bezüglich der Ganglienzellen zunächst eine starke Störung der Reihenanordnung; die einzelnen Elemente bieten verschiedene Formen schwerer Veränderung, unter welchen Schrumpfungen besonders stark hervortreten. Die Markfasern der Rinde sind im Bereiche der schweren Affektion der Meningen bis auf sehr dürftige Reste der Radiärfaserung geschwunden; der Faserfilz im Markgewebe ist stark gelichtet, so daß sich die Markleisten in grauer Farbe präsentieren.

Fs ist nun vom größten Interesse, dem Verhalten der Hirnsubstanz an denjenigen Stellen nachzugehen, wo die meningeale Affektion sich nur in geringem Grade geltend macht; es ist hervorzuheben, daß hier die normalen Beziehungen zwischen Meningen und Gehirn bestehen insofern, als der epicerebrale Raum erhalten ist. Ws ergibt sich der charakteristische Befund der progressiven Paralyse in diffuser Ausbreitung über die ganze Rinde: Gefäßveränderungen mit Plasmazellinfiltrationen, Gliawucherung, Stäbchenzellen, Erkrankung der Ganglienzellen, Markfasernschw und, welcher sich auch hier auf das Marklager erstreckt. An einzelnen Rindenstellen findet man Erscheinungen eines initialen ,,s pongiösen Rindenschwundes".

Kleinhirn: Der Prozeß, welcher die Meningen des Großhirns in der früher geschilderten Weise affiziert hat, erstreckt sich auch auf das Kleinhirn, zeigt aber hier im allgemeinen eine geringere Intensität. In den Furchen finden sich aber zuweilen bedeutende herdartige Verdickungen der meningealen Septen, welche sich tumorartig von der Umgebung abheben und durch das Platzgreifen nekrotischer Vorgänge im Zentrum ein charakteristisches Gepräge erhalten. Riesenzellen fehlen aber hier ebenso wie im Großhirn. 
Auch im Bereiche des Kleinhirns findet man das Hirngewebe an vielen Stellen direkt in Mitleidenschaft gezogen; es findet ein unmittelbarer U̇bergang des meningealen Prozesses auf das Hirngewe be statt, indem die Meningen mit der Gehirnoberfläche verschmelzen und Infiltrationselemente sich über das angrenzende Hirngewebe ergießen. Die ersten Stadien dieser Beeinflussung stellen sich an Schnitten, welche einer Kernfärbung unterzogen wurden, derart dar, daß zunächst eine enorme Zunahme der gliösen Elemente, Bergmannschen Fasern und Gliazellen, sehr häufig in Stäbchenform, in der Molekularschichte zum Vorschein kommt (Tafel XI, Fig. 3). Im Endausgange des Prozesses erscheinen einzelne Windungen und auch ganze Läppchen verkleinert, geschrumpft, aller nervösen Elemente beraubt und von einer abundanten Gliawucherung eingenommen. In dieser Weise repräsentiert sich auch der bei der makroskopischen Beschreibung erwähnte Herd an der Kleinhirnoberfläche.

Wie im Großhirn finden sich auch hier a u Berdem diffuse Veränderungen in der für die progressive Paralyse charakteristischen Form und Verteilung. Außerdem begegnen wir sehr häufig mehrkernige n Purkinjezellen; zur Illustration der Häufigkeit der Mehrkernigkeit sei erwähnt, daß3 in einem Schnitt von $1 \mathrm{~cm}$ im Durchmesser 11 derartige Zellen zu finden sind.

Die meningeale Erkrankung setzt sich auch auf das Rückenmark bis in dessen unterste Partien fort; auch hier macht die Affektion der Meningen ihren Einfluß auf die benachbarte Nervensubstanz geltend, und zwar in erster Linie durch eine starke Verbreiterung der gliösen Randschicht. Neben der in Form eines Ringes auftretenden Randsk le rose findet man herdförmige Ausstrahlungen des Prozesses, myelitische Veränderungen, welche weiter in die Marksubstanz hineinreichen.

Die von den Meningen in die vielfach verbreiterten Gliasepten einstrahlenden Gefäße sind häufig stark infiltriert, und auch in der grauen Substanz sind die Lymphscheiden der Gefäße oft mit Infiltrationszellen vollgestopft; es handelt sich aber fast ausschließlich um $\mathrm{P}^{\prime \prime}$ smazellen.

Eine leichte, beiderseitige l)egeneration des Pyramidensutenstranges gehört offenbar der Paralyse an.

Sehr häufig sind Heterotopien von Ganglienzellen in die weiße Substanz zu finden. Wir wollen nur eine Stelle herausgreifen: im obersten Dorsalmark sehen wir 3 Ganglienzellen an der Einmündungsstelle des Septum longitudinale posterius in die hintere Kommissur; verfolgen wir das Septum gegen die Rückenmarksperipherie zu, so begegnen wir noch einer aus 3 Ganglienzellen bestehenden Gruppe, welche in das Septum eingebettet sind.

Aus dem mikroskopischen Befunde ist noch die lebhafte Ependymwucherung erwähnenswert. - 
Das klinische Bild der bei dem 14jährigen, in seiner körperlichen Entwicklung zurückgebliebenen Mädchen beobachteten Psychose lenkte vom Beginne der Erkrankung das diagnostische Kalkül auf eine progressive Paralyse; diese Annahme wurde durch den nervösen Befund unterstützt. Auch die terminalen Erscheinungen mit den epileptiformen Anfällen traten nicht derart aus dem Rahmen des der progressiven Paralyse zugehörigen Krankheitsbildes, daß man die gestellte Diagnose hätte fallen lassen können. Die konstatierte Höhe der Pleocytose war wohl auffällig, ist aber immerhin auch bei progressiver Paralyse möglich.

Wie in einer großen Zahl der Fälle von juveniler Paralyse standen uns auch hier aus der Anamnese keine Anhaltspunkte für eine Lues der Kranken selbst oder der Ascendenz zu Gebote; deren infantiler Habitus und die Sattelnase ließen aber mit einer gewissen Berechtigung eine hereditäre Syphilis als ätiologisches Moment sowohl für die Entwicklungsstörung als auch für die psychische Erkrankung annehmen. Wie die Sektion ergab, machte sich die Entwicklungshemmung charakteristischerweise in besonders hohem Maße im Bereiche des Genitales geltend, welches auf einer kindlichen Stufe blieb. 1)ie an der Leber nachgewiesenen Einziehungen scheinen ebenfalls auf eine hereditäre Lues hinzuweisen.

Im pathologisch-anatomischen Befunde steht nun zunächst eine in dieser Art zum Bilde der progressiven Paralyse nicht gehörige meningeale Erkrankung im Vordergrunde. Es bestehen aber daneben die typisehen, über das ganze Zentralnervensystem verbreiteten Erscheinungen der progressiven Paralyse; durch die Entwirklungsstörungen im Kleinhirn ist die Erkrankung gleichzeitig als eine auf hereditärer Lues beruhende streng charakterisiert.

Die Affektion der Meningen stellt also eine neben dem chronischen Prozeß der Paralyse verlaufende akutere krkrankung dar. Was die Natur derselben betrifft, so ist nur zwischen Tuberkulose und Syphilis die Entscheidung zu treffen. Für die Tuberkulose fehlen nun sowohl im allgemeinen Befunde, als auch im histologischen Bilde der Meningen alle Anhaltspunkte: es findet sich nirgends in den übrigen Organen ein tuberkulöser Herd, und in den Meningen vermißt man die charakteristischen Bilder der Miliartuberkel; wenn wir uns noch darauf berufen, daß eine sorgfältige Fahndung nach Tuberkelbacillen erfolglos blieb, so können wir Tuberkulose mit gutem Gewissen ausschließen.

Wir haben also eine, in stärkstem Grade an der Gehirnconvexität ausgebildete lue tische Me ningitis vor uns, welche sich auf das Kleinhirn und Rückenmark fortsetzt und das benachbarte Nervengewebe in Mitleidenschaft zicht: eine Meningoencephalitis und Meningomyelitis. Die Affektion trägt in den hauptsächlichsten Zügen die 
Charaktere, welche Alzheimer für diese Erkrankung in scharfer Weise hervorgehoben hat. Die Differenzen, welche sich in unserem Falle gegenüber den Befunden Alzheimers ergeben, sind in vieler Hinsicht offenbar dadurch bedingt, daß hier die luetische Erkrankung auf dem Boden der der Paralyse zugehörigen chronisch-entzündlichen Veränderungen zur Entwicklung kam. Die luetische Affektion scheint zum Schlusse einen besonders akuten Verlauf genommen zu haben und kam in klinischer Hinsicht offenbar erst in den terminalen Erscheinungen zur Äußerung.

Die Kombination von tertiärluetischen Erscheinungen mit juveniler Paralyse, welche allen erwähnten Umständen nach als durch hereditäre Lues bedingt anzusehen ist, bildet eine bemerkenswerte Ergänzung unserer bisher veröffentlichten Reihe von echt-syphilitischen Affektionen bei Paralyse. Bei juveniler Paralyse scheinen spezifisch-luetische Erscheinungen bisher sehr selten nachgewiesen worden zu sein. Bei der Durchsicht der Literatur konnte ich nur eine einzige Beobachtung von Ka plan und Ma ye ${ }^{1}$ ) auffinden; es handelte sich hier um kleine Gummen in den Meningen bei einem klinisch und anatomisch als juvenile Paralyse deklarierten Falle.

In unserer Beobachtung begegnet sich interessanterweise mit dem Nachweise von auf hereditärem Wege akquirierter Lues bedingten spezifischen Erscheinungen ein Befund, welchen wir als charakteristisch für die juvenile Paralyse, insoweit sie auf hereditärer Syphilis beruht, ermittelt haben: die Entwicklungsstörungen im Kleinhirn und Rückenmark.

II. 55jabriger Beamter J. 1), wurde am 4. Februar 1911 mit folgender Anamnese aufgenommen ${ }^{2}$ ):

Scit 2 , Jahren nervös, angstlich, wollte wie allein gehen, suchte unter dem Bette nach Einbrechern, wurde streitsüchtig, zankisch, wegen jeder Kleinigkeit aufgeregt. Im Oktober 1908 hatte man bereits an seiner Dienststelle bemerkt, daß er die Fakturen nicht mohr verlaBlich ausstelle, Rechenfehler mache, es mußte ihm daher eine leichtere Arbeit zugewiesen werden. Sieit den letzten Weihnachten ist er sehr zerstreut, begann durch Einkäufe verschiedener unnätzer Dinge große Ausgaben zu machen. Kaufte in der letzton Zeit neue Kleider und mehrere Kravatten mit der Begründung, daß er ein Graf sei und dementsprechend auftreten müsse, hielt sich aber dabei in seinem Außeren sehr unordentlich. Kurz nach Weihnachten und am 3. Februar erlitt er je einen Anfall von etwa $1 / 2$ Stunde dauernder Siprachlosigkeit. War in der letzten Zeit vorwiegend heiter, sprach sehr vicl, die Mahlzeiten verschlang er hastig und heißhungrig.

War früher immer maßig, in der Lehensführung anstandıg. Ist seit 30 Jahren

1) Allg. Zeitschr. f. Psych. 5y, S. 87.

2) Der Fall ist kurz in der Arbeit von $O$. Fischer ,ZZur Frage der anatomischen Grundlage der Athétose double und der posthemiplegischen Bewegungsstorung überhaupt", diese Zeitschr. 7, '.. 485, erwälınt. 
verheiratet, 3 Jahre nach der Vermählung brachte seine Frau ein totes Kind zur Welt, der im nächsten Jahre geborene Sohn lebt und ist gesund.

Status praesens bei der Aufnahme: Beide Pupillen sehr eng, entrundet, lichtstarr mit guter Konvergenzreaktioni. Facialisinnervation ungleich, beim Sprechen Zuckungen um den rechten Mundwinkel. Fibrillires Zittern der Zunge, Tremor der Hảnde. Der Gang stampfend. bei raschen Wendungen Schwanken. Patellarsehnen reflexe beiderseits lebhaft, schwere, typische S prachstörung. Pleocytose, 49 Zellen im $\mathrm{cmm}$.

Psychisches Verhalten: Fr ist in heiterer Stimmung, spricht viel, er besitze Millionen, werde alles aufkaufen.

In den nächsten Tagen schwankt seine Stimmung zwischen euphorischer Heiterkeit und unmotivierter Weinerlichkeit, bald gewinnt eine manische Lustigkeit, welche deutliche Züge von Demenz an sich tragt, wieder die Oberhand. Im April wird er ruhiger, spater steht eine Stumpfheit mit gelegentlichen Reizbarkeitsausbrüchen im Vordergrunde des klinischen Bildes.

Am 9. November früh fiel es auf, daß der Kranke den Kopf steif nach rechts gedreht hielt und einen stcifen Gang hatte: plotzlich traten Zuckungen in der rechten oberen und unteren Gliedmasse auf. Zu Bett gebracht, liegt er mit nach rechts gewendetem Kopfe da, auch die Augen sind nach rechts gedreht; blasendes Atmen, wobei die rechte Mundhalfte durch die durchtretende Luft aufgestülpt wird, wahrend dielinke geschlossen bleibt. Darauf treten Zuckungen in der rechten oberen und unteren Fxtremitat auf. Die Anfalle wiederholen sich in kurzen Pausen durch mehrere Stunden. Pat. ist benommen, reagiert nicht auf Anrufe, nachmittags treten, allem Anscheine nach unwillkïrliche, ausfahrende Schleuderbewegungen des rechten Armes auf. Gegen Abend ist die rechte obere Extremitat in tonischer Beugestellung, die Finger ebenfalls gebeugt. In der darauffolgenden Nacht ist Pat. sehr unruhig, arbeitet in Bette herum, wühlt die Matratzen auf.

Am nächsten Morgen vollstandig stumpf, reagiert auf keinerlei Reize. Die rechte obere und untere Extremität fallen, wenn sie passiv erhoben werden, schlaff herab. Triceps- und Patellarsehnenreflex rechts gesteigert, links ist der P. S. R. nicht auslósbar. Babinski und Fußphänomen beiderseits negativ.

In der Nacht vom 10. zum 11. November und im Laufe des 11. wieder mehrere Anfälle in der rechten Körperhälfte mit Wendung des Kopfes und der Augen nach rechts, mitunter werden die Anfalle allgemein. Am 12. November morgens jst die rechte Körperseite geläh mt, schlaff, Kopf und Augen nach links gedreht; scheint Gegenstande, wolche vor seiner linken Gesichtsfeldhalfte bewegt werden, eher wahrzunchmen als mit der rechten.

Am 15. November ist er weniger benommen, spricht auf Fragen in paraphatischer, ganz unverstandlicher Weise, zeigt kein siprachverständnis. 20. November treten von neuem rechtsseitige Krampfanfälle auf, welche sich bis zu dem am 21. November 1911 erfolgenden Exitus noch mehrfach wiederholen.

Wie aus der ausführlich wiedergegebenen Krankengeschichte hervorgeht, konnte schon der Anamnese nach kaum ein $Z$ weifel bezüglich der zu stellenden Diagnose obwalten. Bis zum Exitus des Kranken trat weder in den Symtomen noch im Verlauf irgendeine Erscheinung zutage, welche das typische Bild einer progressiven Paralyse getrübt hätte. 
Die Sektion und die mikrosko pische Unters uch ung bestätigte die Diagnose der progressiven Paralyse; sowohl der makroskopische als auch der mikroskopische Befund entspricht in jeder Hinsicht dieser Erkrankung. Es ist wohl nicht notwendig, auf die Details des typischen Befundes einzugehen.

Die Untersuchung hatte aber doch in zweifacher Hinsicht ein unerwartetes Ergebnis: erstens zeigte sich in der Gegend des linken Globus pallid us eine mit breiigen, weißlichen Massen erfüllte Höhle - eine ganz frische Erweichung - und mehrere kleine ältere Erweichungen fanden sich in der gleichen Hemisphäre über das Centrum semiovale Vieussenii verstreut. Die mikroskopische Untersuchung dieser Herde ergibt den gewöhnlichen Befund von teils frischen, teils älteren Erweichungen mit der Besonderheit, daß die die älteren Erweichungen begleitenden Entzündungserscheinungen stärker als gewöhnlich ausgeprägt sind. In der linken Arteria fossae Sylvii wurde makroskopisch ein an der Wand festsitzender Pfropf nachgewiesen, und die mikroskopische Untersuchung deckte hier eine Endarteriitis auf, welche durch eine polsterförmige Wucherung der Intima das Gefäßlumen erheblich verengte; an einer Stelle, an welcher ein Endotheldefekt erkennbar ist, haftet ein den Rest des Lumens verschließender Thrombus. Es muß erwähnt werden, daß degenerative Erscheinungen im Bereiche der Intimaneubildung vollständig fehlen. Als zu dem Befunde der Erweichung gehörig sei noch erwähnt, daß die der erwähnten frischen Erweichung entsprechende Pyramidenbahn eine frische, mit Osmium nachweisbare Degeneration erkennen läßt.

$\mathrm{Z}_{\text {weite }} \mathrm{n}$ fanden sich bei der mikroskopischen Untersuchung die uns aus den früheren Beobachtungen bereits wohlbekannten „,miliaren Gummen“. Sie sind über das Stirn- und Schläfehirn beider Hemisphären verstreut; in der Lokalisation der Gummen in der Gehirnsubstanz bietet der Fall gegenüber allen von uns bisher veröffentlichten Beobachtungen eine Besonderheit: während wir bisher die Gummen immer nur in der Hirnrinde - in dieser wohl in allen Schichten - nachweisen konnten, erseheinen sie diesmal a uch in der Marksubstanz der Hirnwindungen (Tafel XI, Fig. 4).

Zum histologischen Bau dieser Gummen haben wir nach den früher gegebenen ausführlichen Schilderungen kaum mehr etwas Bemerkenswertes beizubringen: es sind miliare Gummen von der Art, wie wir sie im Falle I der Publikation vom Jahre 1906 und in der Beobachtung I der Arbeit vom Jahre 1910 beschrieben haben: Infiltrationsherde von Lymphocyten und Plasmazellen mit starkem Überwiegen der ersteren, in deren Zentrum regressive Veränderungen, nekrotische Prozesse, platzgreifen; in der Zone der Nekrose treten Riesenzellen auf, und in 
der Umgebung der Herde macht sich eine reaktive Wucherung der gliösen Elemente geltend. Tafel XI, Fig. 5 gibt das im Übersichtsbilde in der Marksubstanz sichtbare Gumma in stärkerer Vergrößerung wieder.

An vielen dieser kleinen Neubildungen lassen sich Beziehungen zu Gefäßen nachweisen.

Das Auftreten der Gummen in der Marksubstanz ist deshalb besonders bemerkenswert, da hier eine direkte Abhängigkeit der Gummenvon einer etwaigen meningealen luetischen Affektion von vornherein ausgeschlossen werden kann. Übrigens muß hervorgehoben werden, daß die Veränderungen der Meningen in allen Teilen des Großhirns, also auch in den von der luetisehen Lirkrankung betroffenen Partien in keiner Weise von der typischen Form der für die Paralyse charakteristischen, chronischen Entzündung abweicht. Im Umkreise der Gummen macht sich auch hier ebenso wie in den früher beschriebenen Fällen der Einfluß der Gummen auf die Hirnsubstanz in einer stärkeren Betonung des paralytischen Prozesses geltend.

Ebensowenig wie in unseren äIteren Beobachtungen kam hier die luetische Erkrankung im klinischen Bilde zum Ausdruck; wie wir schon einmal ausgeführt hahen, überdeckt eben die grobe, wenn wir uns so ausdrücken dürfen: diffuse, psychische Störung der Paralyse die etwa durch die lokalisierten kleinen Herdchen bedingten feineren Beeinträchtigungen der psychischen Tätigkeit. Die Destruktion, welche die miliaren, in ziemlich großen Distanzen verstreuten Gummen in der Hirnsubstanz bewirken, ist auch viel zu unbedeutend, als daß etwa gröbere Herderseheinungen im klinischen Bilde als Ausdruck einer lokalisierten Erkrankung die Oberhand gewinnen könnten.

Den allgemeinen Fragen, welche sich an die in den vorstehenden 2 Fällen von neuem nachgewiesene Kombination von progressiver Paralyse und echten syphilitischen Veränderungen knüpfen: der Frage der syphilitischen Pseudoparalyse und der ,Lues cerebri diffusa" haben wir bereits in unseren früheren Veröffentlichungen eine so eingehende Würdigung angedeihen lassen, daß wir der Notwendigkeit enthoben sind, hier des Näheren darauf einzugehen.

Landsbergen und Giljarowsky teilen in vieler Hinsicht unseren Standpunkt nicht; in der Diskussion unserer Anschauungen holen sie ihre Argumente aber in den wesentlichen Punkten aus der Rüstkammor der älteren Ansichten, die wir eben bekämpft haben, um eine Klärung der verworrenen Situation herbeizuführen. Wir müßten unsere damaligen Ausführungen wiederholen, wenn wir in die Diskussion mit den beiden Autoren eintreten wollten.

Die in den letzten Tagen ante exitum bei unserem Patienten beobachteten Symptome, die Krampfanfälle und herdartigen Ausfallserscheinungen wurden der paralytischen Erkrankung zugeschrieben; 
gehören doch die hier beobachteten Erscheinungen in der gleichen Symptomatologie zu den alltäglichen Beobachtungen bei Paralyse! Und doch sind sie in diesem Falle ohne Zweifel durch eine zu der paralytischen Erkrankung hinzugetretene Encephalomalacie hervorgerufen worden, welche also den klinischen Verlauf der Paralyse in folgenschwerer Weise beeinflußte.

Encephalomalacische Prozesse wurden noch in folgenden 3 Fällen nachgewiesen:

III. A. F., 56 jahrige Frau wurde am 18. Februar 1908 der Klinik zugeführt.

Ihr Gatte, welcher sich vor 5 Jahren mit ihr verehelichte, gibt an, daß sie im August 1907 von einer linksweitigen Lah mu ng - Gesicht, Arm und Beine waren vollkommen unbeweglich - heimgesucht wurde, ohne daß eine Bewußtseinsstorung eingetreten ware; nach 3 Woehen war die Lahmung wieder verschwunden. Von dieser Zeit a n machte sich aber in auffallender Weise cine Abnahme dor geistigen Kräfte der Kranken bemerkbar, sie war vergeßlich, verdarb in der Küehe die zubereiteten Speisen, indem sie alles durcheinandermischte; bald hatte sie die Mahlzeiten für mehr Personen vorbereitet, als zu Tische kamen, bald wieder kochte sie viel z.1 wenig. In den letzten 3 Wochen drangte sie fortwahrend aus der Wohnung hinaus, wurde sehr unrein, ließ Kot und Urin unter sich.

1)ie Kranke hat aus früherer Ehe einen jetzt 37 Jahre alten Sohn; (ein mal soll sie abortiert haben.

Die somatische Untersuchung der Kranken bei der Autnahme ergibt: Gesichtsausdruck sehlaff, dement. Pupillen: rechts $2 \mathrm{~mm}$, links $3 \mathrm{~mm}$, beide entrundet; rechte lichtstarr, Lichtreaktion der linken Pupille und die Konvergenzreaktion beider trage. Die $Z$ unge und die grespreizten Finger stark zitternd. Die Kraftleistung der linken oberen Extremität wesentlich abgesehwacht, die Reflexe an den oberen Gliedma Ben ohne Besonderheit. Bauchdeckenreflexe beiderseits fohlend (sehr schlaffe Bauchdecken). Patellarsehnenreflexe beiderscitis sehr schwach, keine sichtbare, nur fühlbare Zuckung des Quadriceps cruris, Achillessehnmenclexe beiderseits fohlend. Gang breitspurig, schwankend, wobei das linke Bein etwas ungeschickter aufgesetzt wird und bei der Vorwätsbewegung nachschleift. S' prache näselnd, stark verwaschen, fast unverstandlich.

Beim Examen ist dio Kranke weinerlich, gibt ihr Alter bald mit 22, bald mit 28 Jahren an, ebenso alt sei ihr Sohn; we ß ihr Geburtsjahr nicht, kennt sich im Orte und der Zeit gar nicht aus. Vorgezeigte Gegenstande bezeichnet sie richtig, weiB auch die Monate in der Reihenfolge aufzuzählen; die einfachsten Rechenaufgaben vermag sie aber nicht zu losen. Die Sichrift ist ganz unleserlich, die mit ausfahrenden, zitternden schriftzügen gemachten Buchstaben gehen über- und durcheinander.

Dieser Zustand erfahrt keine wesentliche Veränderung bis zum Fxitus, welcher nach Entwicklung eines Decubitus unter Temperatursteigerungen und Erscheinungen von Herzschwache am 16. Marz 1908 erfolgt.

Im Einklange mit den klinischen Erscheinungen ergab die patho$\log$ isch-anato $\mathrm{m}$ ische und histolog ische Untersuchung das typische Bild der Paralyse. Den klinischen Symptomen der Hinterstrangerkrankung entsprechend ist eine ziemlich stark ausgeprägte Degeneration des Hinterstrangs nachweisbar. 
In beiden Hemisphären finden sich Erweichungsherde: im rech te n $\mathrm{Nucleus}$ caudatus und im rechten Schläfelappen vor dem Gyrus hippocampi, tief ins Innere des Markes hineinreichend; in der linken Hemisphäre vor dem Kopf des Nucleus caudatus im Centrum semiovale; im vorderen Teile des Balkens.

Es sind Herde von Linsen- und Haselnußgröße und erweisen sich nach ihrem Aussehen und ihrer Konsistenz zum Teil als ganz frische, zum Teil als etwas ältere Erweichungen.

Mit Rücksicht auf die etwa ein halbes Jahr vor der Aufnahme der Kranken in die Klinik aufgetretene linksseitige Lähmung wurde der mikroskopischen Untersuchung des Herdes im rechten Nucle us caudatus besondere Aufmerksamkeit geschenkt: Das nervöse Gewebe des größten Teiles des Kopfes ist zugrunde gegangen und erscheint ersetzt durch gigantisch gewucherte Gliazellen und durch einen Gliafaserfilz. In dem gliösen Gewebe finden sich an vielen Stellen kleinere und größere Lücken, welche durch feine, noch in Proliferation befindliche Bindegewebsbündel durchzogen sind und sehr zahlreiche mit Abbauprodukten beladene, bei Osmiumbehandlung stark geschwärzte Körnchenzellen enthalten.

Wir haben ja keine sicheren Kriterien für die genauere Bestimmung des Alters eines Erweichungsherdes; der vorliegende Befund läßt aber jedenfalls die Möglichkeit zu, daß der Herd vor einem halben Jahre entstanden sei und die linksseitige, innerhalb drei Wochen behobene Lähmung bedingt hätte.

Der mikrosk o pischen Untersuchung wurde außerdem die Arteria fossae Sylvii mit ihren Ästen in der Fissura fossae Sylvii zugeführt und zwar in der Weise, daß der die Gefäße enthaltende Hirnblock in Serienschnitte zerlegt wurde. Dic hier gewonnenen Präparate zeigen eine schwere Erkrankung sowohl des Stammes der Arteria fossae Sylvii (Tafel XI, Fig. 6) wie auch deren Verzweigungen: alle Gefäße weisen eine hochgradrige, die Lumina stark beeinträchtigende Wucherung der Intima auf. Die Me ninge $\mathbf{n}$, in welchen diese Gefäße eingebettet sind, fallen durch die starke hy perplastische Wucherung des Bindegewebes auf; gleichzeitig besteht aber eine hochgradige entzündliche Infiltration des meningealen Gewebes, und insbesondere ist die Adventitia der Gefäße und deren Umgebung von massigen Infiltraten durchsetzt, in welchen Lymphocyten den größeren Anteil haben.

Die oberflächlichen Partien der die Sylvische Furche begrenzenden Hirnteile sind in ihrer Struktur schwer beeinträchtigt; es finden sich in der Rinde Gewebslücken, welche als Folgen alter Hrweichungen anzusprechen sind; sie sind häufig ausgefüllt mit Infiltrationszellen, Lymphocyten und Plasmazellen. Das nervöse Gewebe ist in den oberen Rindenschichten zugrundegegangen und ersetzt durch Gliagewebe. 
IV. M. H., 31 jahrige Frau, wurde am 8 . Auyust 1903 in die Klinik aufgenommen, Exitus am 2. Marz 1906.

Die Kranke, ein uneheliches Kind, dessen Mutter in 68. Lebensjahre an Tuberkulose gestorben ist, hat zweimal geboren, einmal abortiert. Der Abortus fiel zwischen die beiden Geburten, von denen die 2. vor 3 Wochen erfolgte. Während dieser Schwangerschaft sollen sich die ersten Krankheitserscheinungen entwickelt haben: Pat. lachte häufig ohne Grund, war sehr unternehmungslustig, wollte reiten, Reisen unternehmen. Als es zur Geburt kam, glaubte sic Stuhldrang zu haben - plotzlich stürzte das Kind hervor und nur die Dazwischenkunft des Mannes behütete das Kind vor einer Verletzung. Den Tag darauf war sie sehr erregt, wollte das Kind erwürgen, lief nur mit dem Hemd bekleidet zum Hause hinaus. Sie außerte, das Kind gehore nicht ihr, sie sei unglüeklich, sei eine Bettlerin, man solle sie erschlagen. In der letzten Zeit war sie unrein. wollte sich nicht waschen, nicht auskleiden lassen. Ihr Gatte, von welchem diese Anamnese herrührt, kennt die Kranke seit 13 Jahren.

Bei der Aufnahme in die Klinik ist sie in weinerlicher stimmung, bringt absurde Wahnideen depressiven Inhaltes vor: sie sei crschlagen, man habe ihr alles genommen, alle Loute und sie selbst sollen zerhackt werden - man vermißt aber dabei die adaquate Affektbetonung. Sie bietet das Bild einer Verwirrrtheit; ist über Zcit, Ort und die personlichen Verhaltnisse desorientiert. Nach einigen Tagen wird sie klarer, láßt sich zu einfachen hauslichen Arbeiten verwenden; in ihrem ganzen Wesen drückt wich jedoch sehr deutlich eine höhergradige geistige Schwaiche aus. Sie bietet cine ausdruckslose Miene, in der Gesichtsmuskulatur ist ein ausgesprochenes Beben wahrnchmbar. Narh einem Monate glaubt sie bereits $1 / 4$ Jahr hier zu sein, weiß das Datum und das Jahr nicht. kann einfache Rechenaufgaben im Einmaleins nicht losen.

Im Laute des Jahres 1904 wird vic zusehends dementer, ist wrgen ihrer Ungeschicklichkeit und Verweßlichkeit zi gar keiner Arbeit mehr verwendbar, lebt stimpt dahin, ist elphorisch. Dis Siprache wird stolpernd, verwaschen, die Schrift ganz unleserlich.

Vom Marz des Jahres 1905 muß sie zu Bett gehalten werden, da der Gang sehr unsicher wird; sie sch w ankt meist nach rechts hin, stürzt auch haufig zu Boden. Jie Jemenz macht weitere Fortschritte, die Kranke ist vollkommen stumpf, unrein, muß gefüttert worden, die Sprache ist stark bebend, verwaschen, stolpernd, oft ganz und gar unverstandlich.

Was den somatischen Befund betrifft, so war bei der Aufnahme der Kranken auBer 'Tremor der Zunge und der Hände und steigerung der Patellarund Achullessebnenreflexe nichts Benerkenswertes. Die Pupillen zeigten auch weiterhin keine ausgesprorheneren Storungen; hin und wieder eine Differenz in der Pupillenweite.

Ein Befund rom 10. April 1905 lautet: Pupillen mittelweit, linke > rechte. Rechtsseitige Gesichtamuskulatur leicht paretisch. Die Zunge wird unter starkem Beben vorgestreckt und weicht nach rechts ab. Die Finger beim Vorstrecken sehr unruhig, stark zitternd, die Bewegungen der Hande erfolgen langsam und ungeschickt, ruckweise. Dic Kraft der unteren Extremitaten nicht wesentlich abgeschwacht, trotzdem vermag sich die Kranke kaum auf den Beinen zu erhalten, sehwankt nach rechts hin, bei raschen Wendungen fallt sie nach rechts und rückwarts um. Die Patellarsehnenreflexe sehr gesteigert, jaher, stark austahrender Ausschlag, Fußklonus beiderseits, stärker rechts. Babinski negativ.

Am 26. Februar 1906 erscheint die Kranke benommen, blickt fortwithrend krampfhaft nach links hin; es scheint eine rechtsseitige Hemianopsie zu bestehen. 
Die Pat. reagiert auf keine Aufforderungen zu Handlungen, bei spontanen Bewegungen wird der linke Arm bevorzugt. Bauchreflexe beiderseits fehlend. Patellarsehnenreflexe rechts deutlich lebhafter, Achillessehnenreflex dagegen links viel stärker, klonisch. In den nachsten Tagen unregelmäßige Temperatursteigerungen bis auf $39^{\circ}$, Steigerung der Benommenheit. Am 2. März verfällt die Kranke, ist stark benommen, der Puls steigt auf 170, die linke Pupille ist maximal erweitert, bei relativ enger rechter Pupille ( $2 \mathrm{~mm}$ ), beide lichtstarr, die Buldi wandern unter raschen nystactiformen Zuckungen von rechts nach links, langsamer zurück, um dann wieder nach links zu schnellen. Um $10 \mathrm{Uhr}$ abends tritt der Exitus ein, nachdem durch 3 Stunden Cheyne-Stokes bestanden hatte.

Die am 10. April vorgenommene $L u m b a l p u n k t ı n$ ergab einen gesteigerten Druck und reichliche Pleocytose.

Die Sektion ergab im Körperbefund eine chronische Tuberkulose der linken Lungenspitze und der peribronchialen Lymphdrüsen und eine akute Miliartuberkulose.

Das Gehirn ist deutlich atrophisch besonders im Stirnteil, von stark diffus getrübten Meningen bedeckt; an der Gehirnbasis, im Trigonum interpedunculare und im Bereiche der Sylvischen Furche sind die Meningen serös durchtränkt, sulzig infiltriert und mit kleinen grauen Knötchen durchsetzt. Die Ventrikel stark dilatiert, im IV. Ventrikel deutlich hervortretende Ependymgranulationen.

Im linken Nucleus lentiformis befindet sich ein auf den Kopf des Nucleus candatus übergreifender, unregelmäßiger, fast $1 \mathrm{~cm}$ im Durchmesser haltender Erweichungsherd, welcher bereits zu einer Cyste umgewandelt ist.

Die mikroskopische Untersuchung des Gehirns und Rückenmarks ergibt die zweifellosen Symptome einer progressiven Paralyse und einer cerebrospinalen, tuberkulösen Meningitis; was die letztere Erkrankung betrifft, so ist sie durch den Nachweis von Miliartuberkeln und Tuberkelbazillen sichergestellt.

In einem derartigen Falle besteht die von $\mathrm{Nissl}$ und Alzheimer inaugurierte scharfe histologische Diagnostik die Feuerprobe; es ist ein Leichtes, die Gehirnveränderungen, welche der progressiven Paralyse zukommen, von der meningealen tuberkulösen Erkrankung abzuscheiden und entsprechend zu bewerten.

Es sei gleich hier erwähnt, daß die klinische Aufdeckung dieser Komplikation viel größeren Schwierigkeiten begegnete; die diagnostischen Erwägungen waren naturgemäß von dem Gedanken an die bestehende Grundkrankheit der progressiven Paralyse derart beeinflußt, daß die Natur der Komplikation in dieser Richtung gesucht wurde. Nach den klinischen Symptomen lag es demgemäß am nächsten, eine linksseitige Pachymeningitis haemorrhagica anzunehmen. Es wird immer schwierig sein, aus dem vielgestaltigen Bilde der diffusen Hirnerkrankung der progressiven Paralyse eine cerebrale Komplikation richtig einzuschätzen. 
Was nun den Erschein ungsherd betrifft, so bietet er auch nach dem histologischen Befunde die Charaktere eines ,alten" Prozesses. Die Bestimmung des Alters des Herdes entzieht sich einer irgendwie genaueren Beurteilung. Den histologischen Kennzeichen nach könnte seine Entstehung wohl auch in die Zeit vor dem Ausbruche der Paralyse fallen. Eine derartige Annahme verliert aber sehr an Wahrscheinlichkeit, wenn wir uns vor Augen halten, daß es bei der Entstehung des ziemlich großen Erweichungsherdes zur Zeit psychischer Gesundheit nicht ohne eine schwere Kirschütterung des cerebralen Zustandes, welche in den anamnestischen Angaben zum Ausdruck kommen müßte, abgegangen wäre. Viel leichter ist es möglich, daß innerhalb der Paralyse die cerebralen Erscheinungen, welche eine derartige Erweichung begleiten, übersehen werden, da sie cben durch die schweren Symptome der Paralyse maskiert werden.

Wenn wir nun die klinischen Symptome in dieser Richtung einer genaueren Analyse unterziehen, so müssen wir hervorheben, daß bei der Aufnahme der Kranken in die Klinik Herderscheinungen vollkommen fehlten. Im März des Jahres 1905, also etwa ein Jahr vor dem Exitus, machte sich eine sehr erhebliche Unsicherheit des Ganges bemerkbar, wobei die Neigung nach rechts zu fallen besonders auffiel. Fin bald darauf aufgenommener Status deckte noch andere auf die linke Hemisphäre hinweisende Herderscheinungen auf: Die rechtsseitige Gesichtsmuskulatur erwies sich als paretisch, die $Z$ unge wich nach rechts ab und auf der rechten Seite war eine stärkere Ausbildung des FuBklonus nachweisbar.

Es kann ja keinem Zweifel unterliegen, daß diegleichen Symptome auch als Ausdruck der paralytischen Erkrankung an und für sich in Erscheinung treten können. Mit Rücksicht auf den anatomischen Befund haben wir aber eine gewisse Berechtigung, diesem einen Einfluß auf die Gestaltung des klinischen Bildes einzuräumen.

V. F. S. 42jahriger Kutseher, an 15. Dezemder 1901 der psychiatrischen Klink von der propadeutischen Klinik zugeführt.

Seine Frau will seit 6 Monaten bemerkt haben, daßer..nachdenklicher" s.i; gleichzeitis matehte wich eine Eruchnerung tler Siprache geltend, welehe bis in die letzte Zast immer mehr zumahm. Wr schlief mehr als früher und geriet leicht in weinerliche Stimmung. Zweimal und zwar vor 4 und 2 Monaten beobachtete die Frau in der Nacht. daß die sechte Hand und der rechte FuB des Pat. zackte. Vor cinem Monat wurde cr lon cinem etwa 2 Minuten dauernden Schwindel befallen; or legte sich zu liette, am nachsten 'lage ging er aber wjeder sriner Beschaftigung als Kutscher nach; ar arbeitete bis zum 1. Dezember in gewohnter Weise.

Aus dem Sitatus praesens bei der Aufnahme ist hervorzuheben: Pupillen different, rechte < linke, rechte verzogen. Lichtreaktion trager. St arkes Beben der Gesichtsmuskulatur, rechte Nasolabialfalte seschter, der entsprechende Mundwinkel tirfer stehend. Zu ung stark zitternd, wejcht beim Vorstrecken nach rechts 
ab. Patellarsehnenreflexe sehr lebhaft, Andeutung von Fußklonus. Sehr deutliche Sprachstörung im Sinne von Verwaschensein und Silbenstolpern.

Pleocytose positir (21 Zellen im emm), Nonne-A pelt Phase J. positiv. Wassermann im Serum und Liquor positiv.

Psychisch bietet der Kranke dauernd das Bild einer hochgradigen, euphorischen Demenz. Am 5. März 1912 nachmittags treten zwei schwere, linksseitige Krampfanfälle auf, nach denen die Sprache und der Gang eine starke Beeinträchtigung erleiden. Am 15. Mai liegt Pat. zur Zeit der Nachmittagsvisite mit halbgeschlossenen Augen, nach links gedrehte $\mathbf{K}$ o p fe in passiver Rückenlage im Bette, reagiert auf keinerlei Anruf und Reize. Der rech te Arm befindet sich in Beugekontraktur, ist unbeweglich, passiven Bewegungen des linken Irmes setzt er Widerstand entgegen, und hin und wieder werden hier auch spontan Bewegungen ausgeführt. Das rechte Bein wird maximal gestreckt in Spitzfußstellung gehalten, der Patellarsehnenreflex ist hochgradig gesteigert, es besteht sehr lebhafter FuBklonus und Babinski; links Reflexe schwacher, Babınski negativ. Den nächsten Tag über bleibt der Zustand unverandert, Pat. ist benommen, nimmt aber die ihm in den Mund geführte Nah. rung $z$ sich. Die linksseitigen Extremitaten werden aktiv bewegt, rechtsseitig besteht eine Lähmung mit Kontrakturstellung der Extremitaten.

Am 18. Mai steigert sich die Benommenheit zu tiefem Sopor, in der linken Seite ist diuernd ('in grobschlagiger Tremor vorhanden. Die Temperatur steigt auf $39,4{ }^{\circ}$ In der darauffolgenden Nacht stellen sich zweimal schwere linksseitige Krampfanfalle ein und unter starkem Temperaturabfall erfolgt a m 19. Mai morgens der Exitus.

Die Sektion ergibt im allgemeinen Befunde als bemerkenswerte Erscheinung eine Mesaortitis luetica und Atherom mit wandständiger Thrombose in der Aorta ascendens. Die Intima der Aorta ist von zahlreichen, leicht erhabenen, gelblich-grauen, geschwürig zerfallenen Herden durchsetzt. An anderen Stellen ist die Intima leicht eingezogen und bläulich durchschimmernd. An den Geschwüren sitzen hier und da polypöse Efflorescenzen auf.

Was den Hirnbefund betrifft, so fällt vor allem eine starke Schwellung der linken Hemisphäre in die Augen (Textfig. 1); die Meningen sind darüber stark getrübt, verdickt, sehr blutreich und an den Gefäßen finden sich gelbliche Begleitstreifen. Die angeschwollene Hemisphäre fühlt sich weich, schwappend an. Die linke Arteria carotis interna ist total durch einen offenbar vital entstandenen Pfropf verstopft.

Am Durchschnitt der Hemisphäre nach Formolhärtung sieht man das ganze Gebiet der Arteriae cerebri anterior und media erweicht; das Gewebe ist noch nicht zcrfallen, aber ganz weich und schwappend, die Rinde geschwellt, verbreitert und unscharf gegen das Mark abgegrenzt.

Die Rinde der rechten $\mathrm{Homisphäre} \mathrm{ist} \mathrm{deutlich} \mathrm{atrophisch,}$ die Meningen leicht getrübt.

Die mikroskopische Untersuchung dieser Hemisphäre zeigt den gewöhnlichen Befund der progressiven Paralyse.

Das Lumen der linken Carotis interna ist an der Verzweigungsstelle 
vollständig durch ein Blutgerinnsel obturiert, welches seiner histologischen Struktur nach als vital entstanden anzusehen ist. Die Gefäßwand erscheint nur wenig durch eine endarteriitische Auflagerung verändert, so daß nicht nur dic Affektion an der Aorta, sondern auch der lokale Befund zu der Annahme berechtigt, daß es sich um eine e mbolische Verstopfung der Carotis handelt.

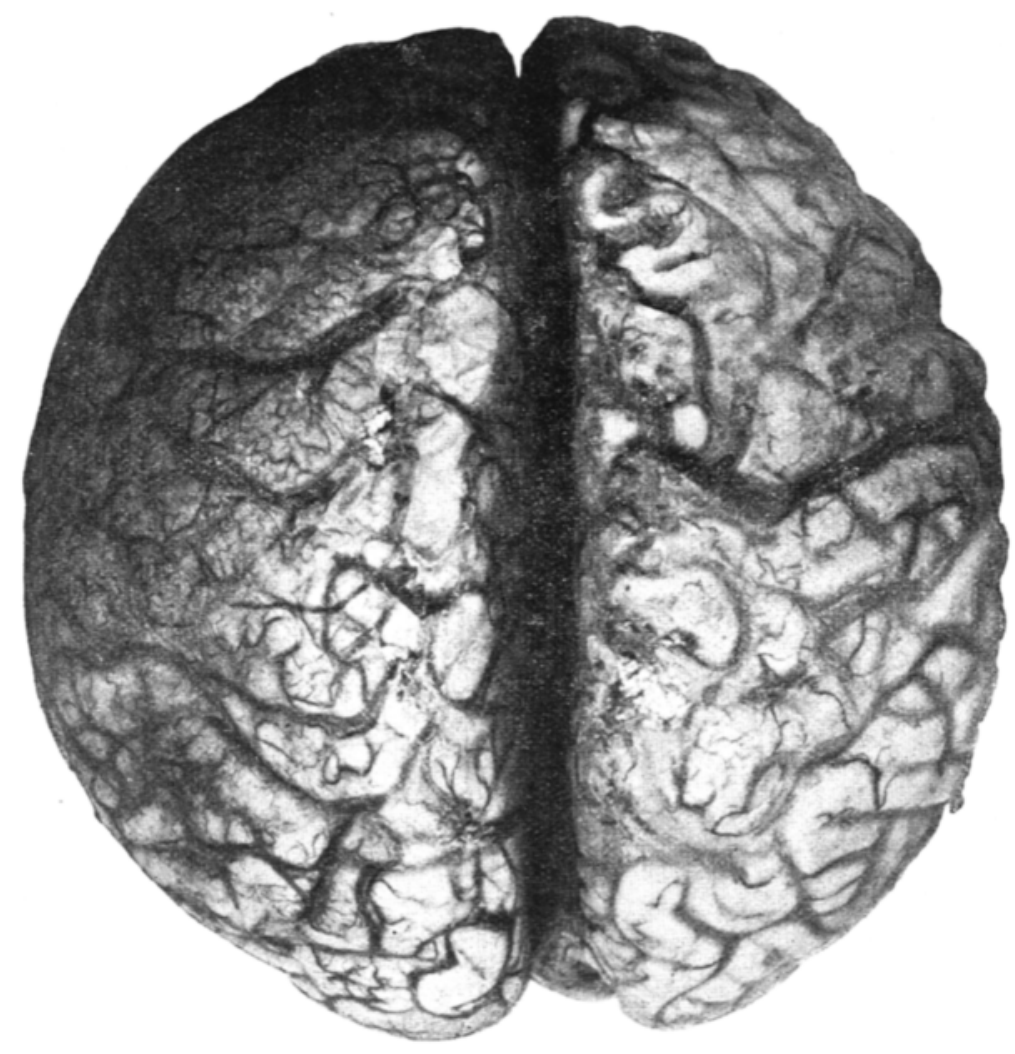

Fig. 1.

Der Fall bietet die seltene Gelegenheit, die histologischen Eigentümlichkeiten eines frischen Erweichungsprozesses - nach dem klinischen Verlaufe war der Exitus etwa $3 \frac{1}{2}$ Tage nach der Embolisierung der Carotis erfolgt - bei einer progressiven Paralyse zu studieren. Es liegt jedoch nicht im Rahmen dieser Arbeit, auf die Histologie der Erweichung des Näheren einzugehen, und es sei daher hier der Befund nur in aller Kürze wiedergegeben: Bei der Betrachtung eines mit Unnas Methylen. blau gefärbten Schnittes, welcher die Meningen, Rinde und Mark enthält, mit schwacher Vergrößerung sieht man den Schichtenbau der Rinde 
in groben Zügen noch erhalten, und auch die Abgrenzung in Rinde und Mark ist ganz deutlich erkennbar. Das sich darbietende Bild der Hirnstruktur erscheint aber etwas verschwommen, wie von einem feinen Schleier bedeckt, durch welchen nur die dunkel gefärbten Kerne diese erscheinen sowohl in den Meningen als auch in der Gehirnsubstanz selbst gegenüber der anderen Hemisphäre bedeutend vermehrt — stärker hervortreten. Der epicerebrale Raum erscheint aufgehoben, die Meningen liegen der Gehirnoberfläche anscheinend fest an und an manchen Stellen verschwimmen infolge Utbertrittes von Infiltrationszellen aus den Meningen auf die Gehirnsubstanz die Grenzen zwischen meningealer Bedeckung und Gehirn.

Stärkere mikroskopische Vergrößerungen ergeben, daß die Verstärkung der Infiltration der Meningen gegenüber der anderen Hemisphäre auf einer Zunahme von Lymphocyten und polynukleären Leukocyten beruht; diese Elemente treten auch auf die Gehirnsubstanz über.

Die Gehirnsubstanz selbst erscheint in ihrem Grundgewebe getrübt, die ektodermalen Elemente, Glia- und Nervenzellen sind gequollen, wie durchfeuchtet, deren Struktur verwischt. Markscheidenpräparate ergeben, daß das Mark der stark geschwollenen markhaltigen Nervenfasern im Zerfall begriffen ist und Schnitte, die nach Bielschowsky behandelt wurden, zeigen die Achsenzylinder nur in kurzen, losen Trümmern. Osmiumpräparate sind naturgemäß überschwemmt von geschwärzten Abbauprodukten.

Ebenso wie die Meningen bieten die Gefäßscheiden eine unverhältnismäßig intensive Infiltration. Sowie aus den Meningen treten aus den Gefäßen Infiltrationszellen in die Gehirnsubstanz, sie ist überschwemmt von mesodermalen Gebilden. Stäbchenzellen treten in ganz auffallend großer Quantität auf.

Neben der Destruktion des ektodermalen Gewebes gibt die Vermehrung und schrankenlosen Ausbreitung mesodermaler Elemente über das Gehirn dem Prozesse das charakteristische Ge präge.

Die Beziehungen des Erweichungsprozesses zum klinischen Verlaufe der Paralyse sind in diesem Falle ganz klar; die Lähmung und Contractur der rechten Halbseite verdankten der zur Paralyse hinzugetretenen Komplikation ihre Entstehung und der Exitus wurde zweifellos durch diese hervorgerufen. Wir müssen bekennen, daß auch hier die Encephalomalacie der klinisch-diagnostischen Feststellung entging, indem die Erscheinungen auf den paralytischen Prozeß selbst als paralytische Anfälle bezogen wurden. -

Die Zusammenstellung und Mitteilung dieser Beobachtungen sollte erweisen, daß Encephalomalacien bei progressiver Paralyse in klinischer und pathologisch-anatomischer Hinsicht eine solche Rolle spielen, daß 
sie der Berücksichtigung wert sind. Sie scheinen nicht viel seltener als die Pachymeningitis haemorrhagica vorzukommen, deren Schilderung insbesondere in der einschlägigen älteren Literatur einen ziemlich großen Raum einnimmt.

In drei Fällen konnten wir die direkten Beziehungen des Erweichungsprozesses zu einer luetischen Erkrankung des Gefäßsystems verfolgen; es liegt nahe, auch in anderen derartigen Beobachtungen der überstandenen Lues einen Einfluß auf die Entwicklung der zuletzt zur Encephalomalacie führenden Erkrankung des Gefäßsystems einzuräumen.

Gegenüber den in der Literatur veröffentlichten Beobachtungen von Erweichungen bei Paralyse aus älterer Zeit, wo der Begriff der Krankheit histologisch keineswegs noch geklärt war, legen wir eine besondere Bedeutung dem Umstande bei, daß die hier verwerteten 170 Fälle histologisch untersucht und in unzweifelhafter Weise als Paralysen deklariert sind.

Für die Genese von stationären Herderscheinungen bei progressiver Paralyse kommen unseren Befunden zufolge neben den herdförmigen Atrophien der „Lissa uerschen Paralyse“ und der Pachymeningitis haemorrhagica auch spezifisch-luetische Prozesse und Encephalomalacien in Betracht. 